\title{
Anticardiolipin Antibodies in Patients with Type 2 Diabetes Mellitus
}

\author{
José María Calvo-Romero, MD and Esther María Lima-Rodríguez, MD
}

Background: There is controversy about an increased prevalence of antiphospholipid antibodies in diabetic patients. The possible implications are little known.

Methods: We prospectively studied all consecutive outpatients with type 2 diabetes mellitus (DM) attended to in an Internal Medicine office. IgM and IgG anticardiolipin antibodies (ACA) were determined by standardized enzyme-linked immunoassay.

Results: Fifty-six patients were included. Only one patient (1.8\%) had a titer of IgM ACA higher than I 5 MPL units and no patient had a titer of IgG ACA higher than 15 GPL units. Six patients (I0.7\%) had low IgMACA titers (4-I5 MPL units) and 18 patients (32.1\%) had low lgG ACA titers (4- I5 GPL units). There were no differences in the frequencies of a low $\lg M$ or $\lg$ G ACA titer or in the means of $\lg M$ and IgG ACA titers in patients with complicated and uncomplicated DM, with and without cardiovascular disease, with and without nephropathy, or with and without retinopathy.

Conclusions: Moderate to high ACA titers must be exceptional in patients with type 2 DM. Low ACA titers may occur in patients with type 2 DM.These low titers do not seem to be associated with complicated DM, cardiovascular disease, nephropathy or retinopathy.

Keywords: Anticardiolipin, Antiphospholipid, Diabetes mellitus.

\begin{abstract}
A complications associated with the presence of antiphospholipid antibodies define the antiphospholipid syndrome. ${ }^{1}$ Anticardiolipin antibodies (ACA) are a subgroup of antiphospholipid antibodies, and the $\mathrm{IgG}$ and IgM isotypes are the most important. ${ }^{1}$ Different abnormalities in diabetes mellitus (DM) configure a hypercoagulable state. ${ }^{2}$ Previous data reflect controversy about an increased prevalence of antiphospholipid antibodies in types 1 and 2 diabetic patients, and the possible implications are little known. ${ }^{3-8}$ We studied the presence of IgG and IgM ACA in a series of patients with type $2 \mathrm{DM}$.
\end{abstract}

\section{Methods}

We prospectively studied all consecutive outpatients with type $2 \mathrm{DM}$ attended to in an Internal Medicine office of a first-level hospital in a rural area from January to July 2006. The population of our area is almost exclusively Caucasian. Patients with diseases associated with ACA (eg, venous thromboembolic disease, other venous thrombosis, conectivopathies) were excluded. ACA (IgG and IgM isotypes) were measured by a previously described standardized enzyme-linked immunoassay (ELISA) method. ${ }^{9}$ The concentration of ACA was measured in MPL or GPL international units (a unit being equivalent to the binding activity of $1 \mathrm{mg}$ of ACA/ml). Positivity of ACA was defined as a titer of IgG ACA higher than 15 GPL units and/or a titer of IgM ACA higher than 15 MPL units, according to the recommendations of our laboratory. All positive results were confirmed, at least, on a second occasion six or more weeks apart. In patients with 2 or more determinations of ACA, we used the mean. 
Table 1. Clinical characteristics of the 56 study subjects.

\begin{tabular}{lc}
\hline Characteristic & $\mathbf{n ~ ( \% )}$ \\
\hline Age (years) & $72.1 \pm 9.9$ \\
Age $>65$ years & $43(76.8)$ \\
Sex female & $32(57.1)$ \\
Complicated DM & $35(62.5)$ \\
Retinopathy & $10(17.9)$ \\
Nephropathy & $18(32.1)$ \\
Estimated GFR $<60 \mathrm{ml} / \mathrm{min} / 1.73 \mathrm{~m}^{2}$ & $12(21.4)$ \\
Albuminuria* & $12(21.4)$ \\
Cardiovascular disease & $24(42.9)$ \\
Ischemic cardiopathy & $5(8.9)$ \\
Cerebrovascular disease & $18(32.1)$ \\
Symptomatic peripheral arteriopathy & $6(10.7)$ \\
Arterial hypertension & $45(80.4)$ \\
Hyperlipidemia & $33(58.9)$ \\
Obesity (BMI $>30 \mathrm{Kg} / \mathrm{m}^{2}$ ) & $12(21.4)$ \\
Insulin therapy & $14(25)$ \\
\hline
\end{tabular}

DM: diabetes mellitus. GFR: glomerular filtration rate.

*Albumin-to-creatinine ratio $>30 \mathrm{mg} / \mathrm{g}$. BMl: body mass index.

Complicated DM was defined as the presence of retinopathy, nephropathy and/or cardiovascular disease. Diabetic nephropathy was defined as an albumin-to-creatinine ratio higher than $30 \mathrm{mg} / \mathrm{g}$ in at least two of three spot urine samples collected within a 6-month period, and/or an estimated glomerular filtration rate (by the MDRD-4 formula) less than $60 \mathrm{ml} / \mathrm{min} / 1.73 \mathrm{~m}^{2}$. Diabetic retinopathy was diagnosed by ophthalmologists including proliferative and non-proliferative retinopathy. Diabetic neuropathy was not studied. Cardiovascular diseases included ischemic cardiopathy (angor or myocardial infarction), cerebrovascular disease (ischemic stroke or transient ischemic attack) and symptomatic peripheral arteriopathy. Symptomatic peripheral arteriopathy was defined as intermittent claudication with an ankle-brachial index less than 0.9 and/or abnormal arterial imaging methods of lower extremities.

We used the chi-square test or Fisher's exact test to compare the categorical variables and the $t$ tests to compare the continuous variables. Statistical significance was defined as a $P<0.05$.

\section{Results}

Fifty-six patients were included and 4 patients were excluded (1 with pulmonary embolism, 1 with primary biliary cirrhosis and 2 with chronic hepatitis C). The clinical characteristics of patients are shown in table 1 . Only one patient $(1.8 \%)$ had a titer of IgM ACA higher than 15 MPL units, and no patient had a titer of IgG ACA higher than 15 GPL units. Six patients (10.7\%) had low IgM ACA titers (4-15 MPL units), and 18 patients (32.1\%) had low IgG ACA titers (4-15 GPL units).

There was no difference in the frequencies of a low $\operatorname{IgM}$ ACA titer (8.6 vs $14.3 \%, P>0.05)$ and a low IgG ACA titer (31.4 vs $33.3 \%, P>0.05)$ in patients with complicated and uncomplicated DM. There was no difference in the frequencies of a low IgM ACA titer $(12.5$ vs $9.4 \%, P>0.05)$ and a low IgG ACA titer ( 37.5 vs $28.1 \%, P>0.05$ ) in patients with and without cardiovascular disease. There was no difference in the frequencies of a low IgM ACA titer ( 0 vs $15.8 \%, P>0.05)$ and a low IgG ACA titer ( 16.7 vs $39.5 \%, P>0.05$ ) in patients with and without nephropathy. There was no difference in the frequencies of a low IgM ACA titer ( 0 vs $13 \%, P>0.05)$ and a low IgG ACA titer ( 50 vs $28.3 \%, P>0.05$ ) in patients with and without retinopathy. The means of IgM ACA and IgG ACA titers in the different subgroups are compared in table 2.

\section{Discussion}

Our study demonstrates, similarly to previous findings, that the presence of moderate to high ACA titers in patients with type 2 DM must be exceptional. In a series of 205 patients with type 1 or type $2 \mathrm{DM}$, no patient had moderate to high ( $>20$ GPL units) IgG ACA titers, and only one had moderate to high IgM ACA titers. ${ }^{3}$ In a series of 46 patients with type 2 diabetes mellitus, no case had an IgG ACA titer higher than $20 \mathrm{GPL}$ units. ${ }^{8}$ On the contrary, in a series of 21 patients with type $2 \mathrm{DM}$, an IgG ACA titer equal to or higher than 15 GPL was present in $9.5 \%$ of cases. ${ }^{6}$

We find low IgG ACA titers (4-15 GPL units) in about onethird of our patients and low IgM ACA titers (4-15 MPL units) in about $10 \%$ of our patients. Another study has described low IgG ACA titers (5-20 GPL units) were significantly more frequent in patients with diabetes with and without cardiovascular disease than in controls (11.4 and 5.6\% vs $0.04 \%$, respectively). ${ }^{3}$ The immunosenescence theory suggests that immune dysfunction with aging is associated with increased autoantibodies production. These autoantibodies may be "innocent bystanders" or may contribute to the pathogenesis of diseases such as atherosclerosis. ${ }^{10}$ The patients of our series were mostly elderly, and the frequency of low ACA titers might be explained by the immunosenescence theory. We did not find statistically significant differences in the frequencies of a low IgM or IgG ACA titer, or in the means of IgM and IgG ACA titers in patients with complicated and uncomplicated type $2 \mathrm{DM}$. Our results suggest that this slight variation in the normal range of ACA titers may be of no clinical importance.

The incidence of IgG, IgM and IgA ACA, anti-beta-2 glycoprotein I antibodies, antiprothrombin antibodies and

Table 2. Comparison of mean ACA titers.

\begin{tabular}{lcc}
\hline & $\begin{array}{c}\text { ACA IgM titer } \\
\text { (MPL units) }\end{array}$ & $\begin{array}{c}\text { ACA IgG titer } \\
\text { (GPL units) }\end{array}$ \\
\hline Complicated DM & $1.2 \pm 3.7$ & $2.1 \pm 3.3$ \\
Uncomplicated DM & $1.2 \pm 3.5$ & $2 \pm 3.1^{*}$ \\
Cardiovascular disease & $1.1 \pm 3.2$ & $2.3 \pm 3.2$ \\
No cardiovascular disease & $1.3 \pm 3.9^{*}$ & $1.8 \pm 3.2^{*}$ \\
Nephropathy & 0 & $1.2 \pm 3$ \\
No nephropathy & $1.8 \pm 4.3^{*}$ & $2.4 \pm 3.2^{*}$ \\
Retinopathy & 0 & $3 \pm 3.2$ \\
No retinopathy & $1.5 \pm 3.9^{*}$ & $1.8 \pm 3.1^{*}$ \\
\hline
\end{tabular}

ACA: anticardiolipin antibodies. DM: diabetes mellitus.

*Non-statistical significance $(P \geq 0.05)$. 
lupus anticoagulant was similar in Chilean diabetic and nondiabetic individuals. ${ }^{5}$ Moreover, no significant correlation was observed between antiphospholipid antibodies and vascular complications. ${ }^{5}$ However, a significantly higher frequency of anti-phospholipid antibodies (IgG or IgM ACA and/ or lupus anticoagulant) has been reported in patients with types 1 and 2 diabetes with macroangiopathy than in patients with uncomplicated diabetes or controls. ${ }^{6}$ We did not find statistically significant differences in the frequencies of a low IgM or IgG ACA titer or in the means of IgM and IgG ACA titers in patients with and without cardiovascular disease.

One study has also described a significantly higher frequency of anti-phospholipid antibodies (IgG or IgM ACA and/or lupus anticoagulant) in patients with types 1 and 2 diabetes with nephropathy than in patients with uncomplicated diabetes or controls. ${ }^{6}$ We did not find statistical differences in the frequencies of a low IgM or IgG ACA titer or in the means of IgM and IgG ACA titers in patients with and without nephropathy or with and without retinopathy. Other studies describe no association between ACA and retinopathy or nephropathy. ${ }^{3,8}$

Our study has several limitations, such as the study design did not include a healthy controls group, the low number of study subjects, and that we were not able to analyze other antiphospholipid antibodies (lupus anticoagulant and anti-beta-2 glycoprotein I antibodies). Moreover, we were not able to study the presence of neuropathy in all patients. The available series investigating the presence of ACA in type 2 DM include a reduced number of cases. Further studies with higher series and prospective follow-up should elucidate the possible role of ACA in type $2 \mathrm{DM}$ and its macrovascular and microvascular complications.

\section{References}

1. Bertolaccini ML, Hughes GR. Antiphospholipid antibody testing: which are most useful for diagnosis? Rheum Dis Clin North Am 2006;32:455-463.

2. Carr ME. Diabetes mellitus: a hypercoagulable state. J Diabetes Complications 2001;15:44-54.

3. Hendra TJ, Baguley E, Harris EN, Khamashta MH, Trembath RC, Hughes GR, Yudkin JS. Anticardiolipin antibody levels in diabetic subjects with and without coronary artery disease. Postgrad Med J 1989;65:140-143.

4. Triolo G, Giardina E, Scarantino G, Seddio G, Bompiani G. Detection of anti-phospholipid (cardiolipin, phosphatidylserine) antibodies in the serum of patients with non insulin-dependent (type 2) diabetes mellitus and macroangiopathy. Coexistence of anti-platelet reactivity. Diabetes Res 1989;10:63-67.

5. Palomo IG, Mujica VE, Alarcón ML, Pereira JG, Vásquez MR. Prevalence of antiphospholipid antibodies is not different in Chilean diabetic patients and normal individuals. J Diabetes Complications 2005; 19:133-137

6. Galtier-Dereure F, Biron C, Vies M, Bourgeois V, Schved JF, Bringer J. Vascular complications of diabetes mellitus: what role for phospholipid-binding antibodies? Lupus 1998;7: 469-474.
7. Gargiulo P, Goldberg J, Romani B, Schiaffini R, Ciampalini P, Faulk WP, McIntyre JA. Qualitative and quantitative studies of autoantibodies to phospholipids in diabetes mellitus. Clin Exp Immunol 1999;118:30-34.

8. Tarkun I, Hacihanefioğlu A, Tarkun P, Cetinarslan B, Cantürk Z. Anticardiolipin and anti-beta2 glycoprotein I antibody concentrations in patients with type 2 diabetes mellitus. Diabetes Res Clin Pract 2005;68:181-187.

9. Hunt JE, McNeil HP, Morgan GJ, Crameri RM, Krillis SA. Antiphospholipid $\beta 2$-glycoprotein I complex is an antigen for anticardiolipin antibodies occurring in autoimmune disease but not with infection. Lupus 1992;1:75-81.

10. Liang KP, Gabriel SE. Autoantibodies: innocent bystander or key player in immunosenescence and atherosclerosis? J Rheumatol 2007;34:1203-1207.

\section{Author Affiliations}

José María Calvo-Romero, MD

Internal Medicine Service

Hospital Ciudad de Coria

Coria (Cáceres)

Spain

Esther María Lima-Rodríguez, MD

Family Medicine

Montehermoso (Cáceres)

Spain 